\title{
On the semantics of temporal when-clauses*
}

\author{
David P. Hall \\ University of California, San Diego \\ Ivano Caponigro \\ University of California, San Diego
}

\begin{abstract}
This paper is about non-interrogative temporal embedded clauses introduced by when (temporal when-clauses), their semantic interpretation and their syntax/semantics mapping. Our goal is to provide a fully compositional account of temporal when-clauses that accounts for their formal identity with interrogative clauses and their difference in meaning. The main idea is that temporal when-clauses are syntactically and semantically free relative clauses. Previous syntactic analyses (Grimshaw 1977, Bresnan \& Grimshaw 1978, a.o.) have provided robust support to the syntactic side of this claim. On the other hand, the semantic proposals for temporal when-clauses that we are aware of (Bonomi 1997, Vikner 2004, Moens $\&$ Steedman 1988) have ignored these syntactic conclusions and have argued for analyses that are problematic for the syntactic/semantic mapping. These semantic analyses are also not fully adequate in handling the interpretative properties of temporal when-clauses. We provide evidence from the distributional and interpretive properties of when-clauses as well as from the temporal alignment of the matrix clause with the when-clause that supports our analysis.
\end{abstract}

Keywords: free relatives, when, temporal clauses, adjuncts

\section{Introduction}

English makes use of various kinds of embedded clauses to convey different temporal relationships between the event described by the matrix clause and the event described by the embedded clause. Some examples are given in in (1)-(6).

(1) I left [when Bill left].

(2) I left [after Bill left].

(3) I left [before Bill left].

(4) I left [while Bill was sleeping].

(5) I have been awake [since Bill left].

(6) I slept [until Bill woke me up].

* We thank the audiences of SALT 20, CUSP 2009, MOSS 2009, and the participants of SemBabble and Language and Brain Lab at UCSD for discussions that have improved this work. 
On the semantics of temporal when-clauses

Among the bracketed embedded clauses in (1)-(6), the one in (1) stands out for at least one reason. Though all the embedded sentences are introduced by a special element (in bold), only the embedded clause in (1) is introduced by a $w h$-word (when) and looks identical to an embedded wh-interrogative clause, as shown in (7).

$$
\text { I wonder [when Bill left]. }
$$

Though virtually identical, the embedded clauses in (1) and (7) receive very different interpretations, due to the different matrix predicates that select them as their complement. The embedded clause in (7) can only be paraphrased with another wh-clause like at what time did Bill leave, while the embedded clause in (1) can be easily paraphrased by a PP containing a headed relative clause like at the time Bill left.

This paper is about non-interrogative temporal embedded clauses introduced by when (henceforth, temporal when-clauses) like (1), their semantic interpretation and their syntax/semantics mapping. Our goal is to provide a fully compositional account of temporal when-clauses that accounts for their formal identity with interrogative clauses and their difference in meaning. The main idea is that temporal when-clauses are syntactically and semantically free relative clauses. Previous syntactic analyses (Grimshaw 1977, Bresnan \& Grimshaw 1978, a.o.) have provided robust support to the syntactic side of this claim. On the other hand, the semantic proposals for temporal when-clauses that we are aware of (Bonomi 1997, Vikner 2004, Moens $\&$ Steedman 1988) have ignored these syntactic conclusions and have argued for analyses that are problematic for the syntactic/semantic mapping. These semantic analyses are also not fully adequate in handling the interpretative properties of temporal when-clauses, as we will discuss in the next section. Non-interrogative embedded clauses introduced by when can also be used with a meaning closer to conditional clauses rather than to temporal clauses, as shown in (8)-(10) and the paraphrases below each example.

[When it rains], I feel sad. cf. If it rains, I feel sad.

(9) [When a natural number is divisible by 2], then the number is even. cf. If a natural number is divisible by 2 , then the number is even.

(10) [When a kid likes a toy], she wants it all the time. cf. If a kid likes a toy, she wants it all the time.

"Conditional" when-clauses will not be dealt with in this paper - though we hope that future work will allow us to extend our proposal to them as well - and the reader is referred to Farkas \& Sugioka's (1983) insightful work.

The paper is organized as follows. Section 2 briefly discusses the previous syntactic and semantic treatments of temporal when-clauses, illustrating the radical 
divergence of the two, and some shortcomings of the previous semantic approaches. Section 3 presents our proposal for uniting the syntax and semantics of these clauses and a fully compositional semantic derivation is discussed in detail. Section 4 further discusses and motivates the two silent prepositions utilized in our analysis. Section 5 provides arguments for the presence of a gap in temporal when-clauses. Section 6 illustrates the nominal nature of these clauses. Section 7 provides further evidence for the free relative analysis from the temporal alignment of the when-clause and the matrix clause. Section 8 discusses the issue of the ambiguity of temporal whenclauses between referring to a time interval and an event. Section 9 briefly touches upon temporal when-clauses that are not interpreted as referential. Finally, section 10 concludes.

\section{Previous syntactic and semantic approaches}

Since the 1970s, syntacticians have treated temporal when-clauses as free-relatives, i.e., non-interrogative embedded clauses introduced by $w h$-words that have a gap and have the same distribution as DPs or PPs (Grimshaw 1977, Bresnan \& Grimshaw 1978, a.o). As such, they fit into the paradigm in (11)-(15) below where the wh-word is shown in bold and the gap is indicated by an underline. The paraphrases next to each example show that free relatives can always be replaced with DPs or PPs.

(11) I ate [what you cooked_ ]. "I ate [the things that you cooked]."

(12) I'll marry [who you choose_ ]. "I'll marry [the person that you choose]."

(13) I sat [where the sun shined_ ]. "I sat [in the place that the sun shined]."

(14) I skied [how you taught me_ ]. "I skied [in the manner that you taught me]."

(15) I drank [when Steve drank _ ]. "I drank [at the time that Steve drank]."

We fully endorse the syntactic analysis of temporal when-clauses as free relatives. Therefore, temporal when-clauses should exhibit the same semantic properties as other free relatives as well, in particular they should be interpreted as DPs or PPs. In the next sections, we show that this prediction is borne out.

On the other hand, the semantic analyses of temporal when-clauses that we are aware of (Bonomi 1997, Vikner 2004, Moens \& Steedman 1988) have treated temporal when-clauses as gapless clauses that occur as one of two arguments of the temporal operator introduced by when. According to these analyses, the wh-word when semantically behaves as an operator taking two clauses/events as its arguments and returning true if the clauses/events have the temporal relation of "overlap".

Bonomi's (1997) definition of when is reproduced in (16) below. It takes two events (e and e') and returns true just in case the temporal relation of overlap holds 
On the semantics of temporal when-clauses

between them. Vikner's (2004) definition of when is similar in form and spirit.

$$
\begin{aligned}
& \text { when }(\mathrm{A}, \mathrm{B}) \sim>\lambda \mathrm{e} \lambda \mathrm{C}\left[\mathrm{A}(\mathrm{e}) \wedge \forall \mathrm{e}^{\prime}\left[\mathrm{C}\left(\mathrm{e}^{\prime}\right) \leftrightarrow \mathrm{B}\left(\mathrm{e}^{\prime}\right) \gg \ll\left(\mathrm{e}, \mathrm{e}^{\prime}\right)\right]\right] \\
& \gg \ll \text { indicates "overlap" }
\end{aligned}
$$

This approach to temporal when-clauses raises several problems. First, the $w h$-word when and the wh-clause it introduces are treated as syntactically and semantically very different from all the other $w h$-words and $w h$-clauses, despite the strong evidence supporting the opposite conclusion. For instance, temporal when-clauses are assumed to convey a proposition/event in the very same way as the matrix clause. But temporal when-clauses can be replaced and paraphrased with DPs (17) or PPs (18), as we will discuss further in section 6.

(17) I really hated [when John lied like that $] /[D P$ the time(s) John lied like that].

(18) I left [when Bill arrived $] /[P P$ at the time Bill arrived].

The very same pattern is observed with FRs introduced by where and how:

(19) I really hated [where the banquet was held]/[ $D_{D}$ the place the banquet was held].

(20) I slept [where the shadow was darkest $] /\left[{ }_{P P}\right.$ in the place the shadow was darkest].

(21) I really hated [how he sang that song] $] /[D P$ the way he sang that song].

(22) I recited the poem [how I was taught] / [ $P P$ in the manner I was taught].

Second, since when is not analyzed as a wh-word undergoing movement and leaving a trace, no island-effects are expected. This prediction is incorrect, since temporal when-clauses exhibit interpretative restrictions that can be easily accounted for if island effects are assumed, as we show in section 5.

Finally, the temporal operator analysis incorrectly predicts that temporal arguments should freely occur inside the when-clause. However, this prediction is not borne out. Prepositional temporal phrases cannot co-occur with the when-clauses with normal intonation, that is, without a pause or an intonational break right before the PP, as this (23) would constitute a "filled-gap" situation analogous to (24) below.

$$
\begin{aligned}
& \text { *I read [when you recommended at 5pm ]. } \\
& \text { *I read [what you recommended the book]. }
\end{aligned}
$$

In this way, previous semantic analyses inadequately account for the distributional and semantic properties of when-clauses. 


\section{Our semantic proposal}

We argue that temporal when-clauses are syntactically and semantically free relatives. Syntactically, they are $w h$-clauses that have a gap and the same distribution as DPs or PPs, like all other free relatives. Semantically, like all other free relatives, they denote the maximal element of a given set (Jacobson 1995, Caponigro 2003, 2004, a.o.). In the case of temporal when-clauses, the given set is a set of time intervals or events. The meaning of the $w h$-word when is of the same kind as all other phrasal $w h$-words - a set restrictor. It applies to a set of entities and returns a subset that only contains time intervals or events.

We present the details of our proposal by looking at two different examples of temporal when-clauses, providing their fully detailed semantic derivations, and commenting on them.

The sentence in (25) contains a temporal when-clause that behaves like a PP adjunct of the matrix clause. Its semantic derivation is given in (26). The crucial steps are highlighted in bold. Comments follow. ${ }^{1}$

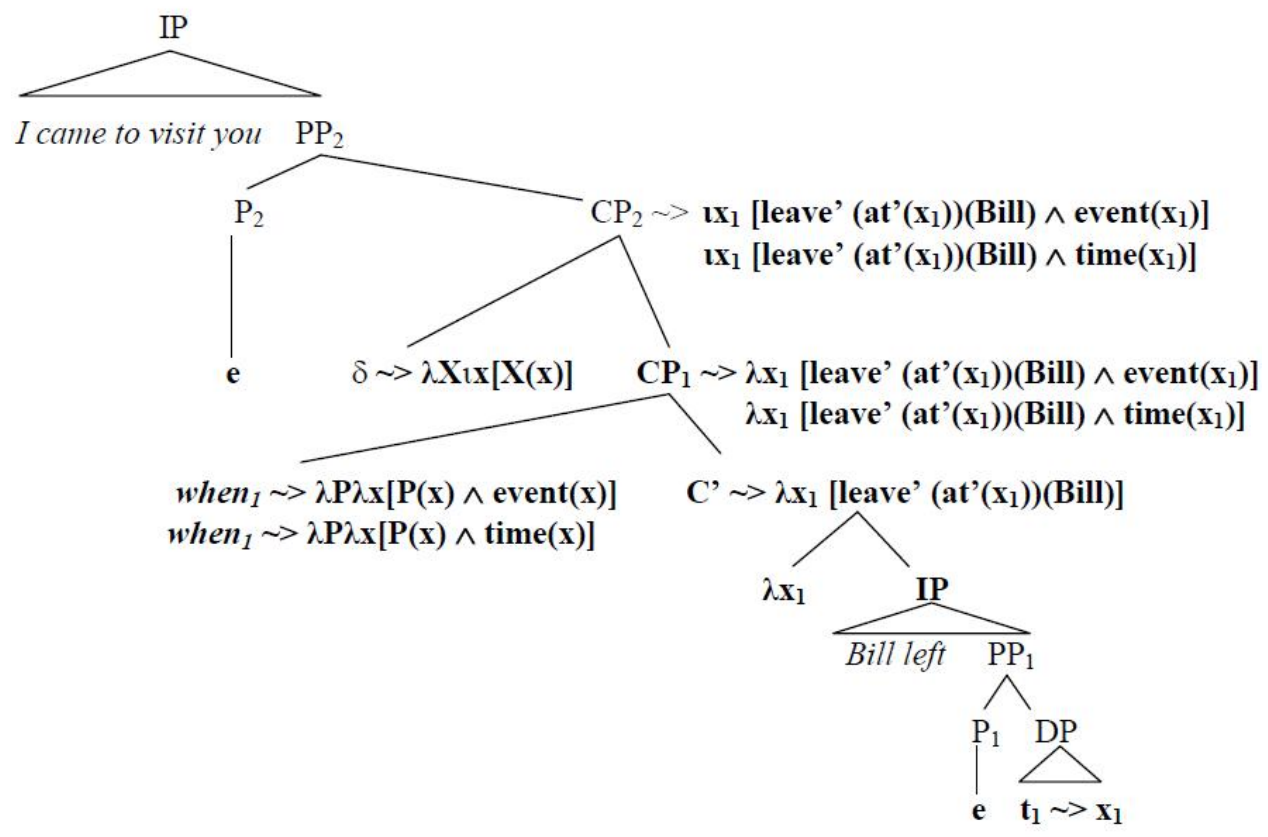

Starting from the bottom of the tree/derivation in (26), the movement of the wh-word when from within the PP adjunct to $\mathrm{SpecCP}_{1}$ leaves behind a trace/variable

1 The syntax of free relatives is an open issue. See van Riemsdijk 2005 for a recent thorough survey of the relevant facts and related issues. 
$\left(\mathrm{t} / \mathrm{x}_{1}\right)$ as for any other $w h$-word. Notice that we are assuming that the $w h$-word when is syntactically a DP that is always base-generated as the complement of a silent preposition $\mathrm{P}_{1}$. We return to this assumption and its supporting evidence in the next section. Standard lambda-abstraction (which we locate in the head C for convenience) applies over the variable, generating the set of entities that are in a certain relationship with Bill's leaving (denotation of C'). Like all other wh-words, when is semantically analyzed as a set restrictor. It applies to a set of entities and returns those that are time intervals (27a) or events (27b).

$$
\begin{aligned}
& \text { a. } \quad \text { when } \sim>\lambda \mathrm{P} \lambda \mathrm{x}[\mathrm{P}(\mathrm{x}) \wedge \operatorname{time}(\mathrm{x})] \\
& \text { b. } \quad \text { when } \sim>\lambda \mathrm{P} \lambda \mathrm{x}[\mathrm{P}(\mathrm{x}) \wedge \operatorname{event}(\mathrm{x})]
\end{aligned}
$$

In the derivation in (26), when restricts the set of entities that are in a certain relationship with Bill's leaving to the subset of those time intervals or events when Bill left. We further discuss the dual nature of this restriction (time intervals and events) and the assumption that when is ambiguous in section 8 .

$\mathrm{CP}_{1}$ denotes a set of entities. The silent preposition $\mathrm{P}_{2}$ that we are assuming takes the when-clause as its complement (we return to this assumption in the next section) selects for an individual denoting expression. A type-mismatch arises. We follow Caponigro $(2003,2004)$ in assuming that a silent syntactic operator $\delta$ encodes the type-shifter $\imath$. When $\imath$ applies to the denotation of $\mathrm{CP}_{1}$ (i.e. the set of time intervals or events when Bill left), it returns the maximal element of that set as the denotation of $\mathrm{CP}_{2}$. The maximal element is the only time interval or event when Bill left, if the set is a singleton, or the plural individual resulting from the sum (in the sense of Link 1983) of all the time intervals or events when Bill left.

We now briefly look at an example that shows the other possible role of a temporal when-clause within its matrix sentence - as an argument. The sentence in (28) contains a temporal when-clause that is identical in form to the one we just discussed in (26), but it now occurs as the object of the matrix predicate. Its semantic derivation is identical to (26) up to $\mathrm{CP}_{2}$.

$$
\text { I hated [when Bill left]. }
$$

The $\mathrm{CP}_{2}$ of the temporal when-clause in (28) denotes the very same individual - the sum of all time intervals or events when Bill left. Since the temporal when-clause now occurs in the object position of the matrix predicate hated, which selects for an individual-denoting complement, a silent $\mathrm{P}_{2}$ is no longer licensed and the $\mathrm{CP}_{2}$ of the temporal when-clause can combine directly with the matrix predicate.

In conclusion, the analysis we just presented treats temporal when-clauses as referential expressions denoting a maximal time interval or event. They can combine with the matrix clause in two ways: either directly as one of the arguments of the matrix predicate or via a possibly silent preposition as a PP adjunct. This is very 
different from previous approaches. They assign temporal when-clauses the same basic propositional nature as their matrix clauses. The wh-word when temporally connects one proposition to the other.

In the next sections, we discuss further details and assumptions of the analysis we just presented and bring further supporting evidence.

\section{Silent prepositions}

Our syntactic/semantic analysis above relies on two silent prepositions: $\mathrm{P}_{1}$, which is the sister of the base-generated position of the wh-word when, and $\mathrm{P}_{2}$, which is the sister of the whole temporal when-clause. In this section, we discuss each of them in turn.

As for $\mathrm{P}_{1}$, we are assuming that when is always base-generated as its DP and leaves a DP trace/variable after moving, because we want a set of entities to be generated as the result of lambda-abstraction. Therefore, the trace/variable over which abstraction occurs has to be a DP of type e. On the other hand, the trace of when does not exactly have the distribution of a standard DP. If anything, its distribution resembles an adjunct PP. Therefore, we assume that when is always base-generated as the complement of a silent P. This is not an ad hoc assumption motivated just by the need to get the right semantic results. Other expressions that are DPs without any doubt have independently received an identical analysis. Emonds $(1976,1987)$ and McCawley $(1988)^{2}$ argue that silent prepositions are needed to account for the behavior of adverbial nominals like the other day (temporal), nice places (locative), and that way (manner), as shown in (29)-(31). They argue that such nominals are always generated as complement of a silent $\mathrm{P}$ when no overt $\mathrm{P}$ occurs as their sister.

I left $[P$ e] $[D P$ the other day].

He went $\left[{ }_{P} \mathbf{e}\right][D P$ nice places].

I like to dance $\left.{ }_{P} \mathbf{e}\right][D P$ that way].

Caponigro \& Pearl (2008) make a similar point for free relatives introduced by where (locative) and how (manner), as shown by the silent preposition e in (32) and (33).

(32) I hated [where I used live $[[P \mathbf{e}] \mathbf{t}]]$.

(33) I liked [how she danced $\left.\left[\left[{ }_{P} \mathbf{e}\right] \mathbf{t}\right]\right]$.

As for $\mathrm{P}_{2}$, the other silent preposition that we made use of in our analysis in (26), it occurs as the sister of the whole temporal when-clause. Unlike the lower silent $\mathrm{P}_{1}$

2 See also Larson 1985 for a different analysis. 
On the semantics of temporal when-clauses

in (26), $\mathrm{P}_{2}$ is not obligatory and its presence depends on the matrix clause. In (26), repeated below as (34), $\mathrm{P}_{2}$ is needed since the matrix VP (come to visit you) does not allow for any other argument and the whole temporal when-clause behaves like a temporal adjunct of the matrix clause. But a temporal when-clause can occur as the complement of a verb like like too, as shown in (35). In this case, only the silent $\mathrm{P}_{1}$ within the temporal when-clause occurs.

I came to visit you $[P 2 \mathbf{e}]$ [when Bill left $\left.\left[\left[_{P 1} \mathbf{e}\right] \mathbf{t}\right]\right]$.

I liked [when you smiled at me $\left.\left[\left[P_{1} \mathbf{e}\right] \mathbf{t}\right]\right]$.

Support for our assumption about the presence of $\mathrm{P}_{2}$ comes from the fact that such a $\mathrm{P}$ can be overt sometimes, as shown by the prepositions from and to in (36). It is not the case that any overt P can take a temporal when-clause as its complement, though, as shown in (37) and (38). This restriction may be due to the recoverability of the content of the preposition from other elements in the sentence.

(36) I lived in Spain [P2 from ] [when I was five $\left.\left[\left[P_{1} \mathrm{e}\right] \mathrm{t}\right]\right][P 2$ to $]$ [when I was seven $[[P 1 \mathrm{e}] \mathrm{t}]]$.

I lived in Spain $[P 2 *\{\mathbf{d u r i n g / o n / i n / a t}\}]$ [when I was five $[[P 1 \mathrm{e}] \mathrm{t}]]$.

I left the party [P2 $*\{$ during/on/in/at $\}$ ] [when the band stopped playing].

Interestingly, the very same pattern is observed with free relatives introduced by where (39)-(40). This analogy brings further support not only to our assumption about $\mathrm{P}_{2}$ but also our analysis of temporal when-clauses as free relatives.

(39) I am going to [where the birds go for winter].

(40) I sat down *\{on/in/at $\}$ [where the chair used to be].

In conclusion, the silent prepositions that we are assuming for our analysis of temporal when-clauses have been independently argued for other constructions that are syntactically and/or semantically similar to ours, like adverbial nominals and free relatives introduced by where or how.

\section{Island effects in temporal when-clauses}

According to our proposal, temporal when-clauses are free relatives, i.e. embedded clauses to which wh-movement has applied. As such, sensitivity to movement constraints, islands in particular, is predicted. In this section, we show that this prediction is borne out.

The sentence in (41) contains two levels of embedding - an embedded CP containing another $\mathrm{CP}$. The $w h$-word occurs in the specifier of the higher $\mathrm{CP}$, but it 
could have been base-generated within either CP. (42) shows the option in which when has been base-generated within the highest $\mathrm{CP}$, as shown by its trace $\mathrm{t}_{1}$. The interpretation that is associated with this structure is such that my eating dinner and Maria's thinking occurred at the same time. (43) shows the other structural option. The $w h$-word is base-generated inside the lower CP, where the trace $\mathrm{t}_{1}$ occurs. The interpretation associated with this structure is such that my eating dinner coincides with the time that Maria suggested as my dinner time.

(41) I ate dinner [ $C P$ when Mary thought [ $C P$ that I should eat dinner]].

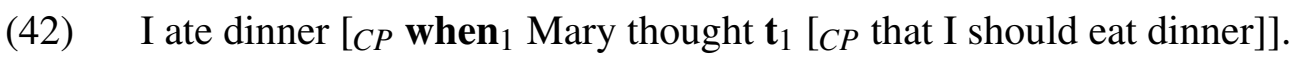
Paraphrase: I ate dinner at a certain time and at that very same time Mary had the thought that I should eat dinner (at some later time).

(43) I ate dinner [ ${ }_{C P}$ when $_{1}$ Mary thought $\left[{ }_{C P}\right.$ that I should eat dinner $\left.\left.\mathbf{t}_{1}\right]\right]$. Paraphrase: I ate dinner at a certain time and Mary had previously had the thought that I should eat dinner exactly at the time I did.

If we change the example in (41) above slightly, in such a way that the lower CP is now embedded within a DP like the suggestion, we create what is called a complex NP/DP island (44). No movement can occur out of it. Therefore, the prediction is that when can only be base-generated within the higher $\mathrm{CP}$ and the whole sentence can only be interpreted as meaning that my eating dinner and Maria's suggestion happened at the same time, as shown in (45). When cannot be based-generated within the lower CP since it would have to move out of an island (46). Therefore, (44) is predicted not to mean that my eating dinner coincided with the time that Maria suggested as my dinner time. The prediction is borne out.

(44) I ate dinner [ $C P$ when Mary made the suggestion [ $C P$ that I should eat dinner]].

(45) I ate dinner $\left[C P\right.$ when ${ }_{1}$ Mary made $\mathbf{t}_{1}\left[D P\right.$ the suggestion $\left[{ }_{C P}\right.$ that I should eat dinner]]].

Paraphrase: I ate dinner at a certain time and at that very same time Mary made the suggestion that I should eat dinner (at some later time).

(46) $*$ I ate dinner $\left[C P\right.$ when ${ }_{1}$ Mary made $\left[D P\right.$ the suggestion $\left[{ }_{C P}\right.$ that I should eat dinner $\left.\left.\mathbf{t}_{1}\right]\right]$ ].

Cannot mean: I ate dinner at a certain time and Mary had previously made the suggestion that I should eat dinner exactly at the time I did.

We just showed that the pattern above can be easily accounted for by our proposal that temporal when-clauses are free relatives involving $w h$-movement. This further supports our analysis. On the other hand, previous semantic proposals would face serious problems in giving a principled account of this pattern since they are assuming no movement takes place within temporal when-clauses. 
On the semantics of temporal when-clauses

\section{When-clauses as DPs and PPs: distribution and interpretation}

Temporal when-clauses have the same distribution as and can always be paraphrased with DPs or PPs (Grimshaw 1977; Declerck 1997). We already saw several examples of PP-like behavior of temporal when-clauses. We give some examples of DP-like behavior in (47)-(49).

(47) I really couldn't stand [when you cried like that $] /[D P$ those situations].

(48) $\quad[$ When Jane arrived $] /[D P$ The moment Jane arrived] was perfect.

$$
\text { It was perfect [when Jane arrived }] /[D P \text { the moment Jane arrived]. }
$$

The very same DP-like and PP-like behavior is observed with free relatives introduced by where and how, as shown earlier in (19) and (20) above. Our analysis captures these facts by analyzing temporal when-clauses as free relatives. As such, they denote an individual like referential DPs, or if they occur as the sister of a possibly silent $\mathrm{P}$, they semantically behave like a PP with a referential DP complement, as we discussed in section 2. This distributional and interpretative pattern is problematic for the previous semantic analyses of temporal when-clauses, though, since they treat temporal when-clauses as propositional in nature.

In the remainder of this section, we briefly review other properties that temporal when-clauses share with DPs and PPs as further support to our analysis.

First, temporal when-clauses can also appear as the complement of the preposition against, as shown in (50).

(50) Compare when you arrived late against [ $D P$ when I arrived early].

Unlike many other prepositions, against does not take clausal complements, but only DP/NP complements (Grimshaw 1977; Declerck 1997).

Second, temporal when-clauses can be conjoined by the conjunctions and or or with DPs like the day or March 1st (51) or PPs like on August 21st or at 3:30 pm (52).

(51) John likes [the day he gets paid/March 1st] or [when I give him money].

(52) The company changed hands [on August 21st /at 3:30pm] and [when it filed for bankruptcy].

On the semantic side, there is also evidence that when-clauses are referential denoting expressions. The evidence comes from the fact that when-clauses can serve as the antecedent of an individual denoting expression like a pro-form or a demonstrative. Again, the temporal operator analyses do not predict this behavior. In (53) below, the pro-form then refers to when I went to Coronado beach, as does 
the demonstrative that. The utterance in (54) also illustrates that then can refer back to the referent introduced by when Bill left.

(53) I got a sunburn [when I went to Coronado Beach $]_{1}$.

Sue lived in Europe then 1 . / That 1 was July 20, 2003.

I came to visit you [when Bill left $]_{1}$. John visited then ${ }_{1}$, too.

These distributional and interpretative behaviors strengthen our analysis of temporal when-clauses as free relatives. Like free relatives, they behave like DPs and PPs syntactically and refer to individuals semantically.

\section{Further evidence: temporal alignment}

Our proposal treats temporal when-clauses as free relatives and the meaning of the $w h$-word when is just a set restrictor, playing no role in determining the temporal alignment between the matrix clause and the temporal when-clause. Rather the two crucial factors that are responsible for such an alignment according to us are (i) the silent or overt preposition that mediates between the two clauses, and (ii) the aspectual nature of the predicates. On the other hand, the previous semantic analyses build the temporal alignment ("overlap") of the two clauses into the definition of when. The facts presented below bring further support to our proposal by showing that the temporal alignment cannot be due to when and thus should not be part of its meaning. The strategy we follow in each case is to show that the very same temporal alignment facts hold even if the temporal when-clause is replaced with a relative clause headed by a temporal nominal and without any $w h$-word. Therefore, the temporal alignment cannot be due to the wh-word when.

The sentences in (55)-(57) all have the same matrix clause, but they differ in their bracketed constituent. (55) contains a temporal when-clause, (56) a complex DP with a relative clause headed by the temporal nominal year, and (57) the very same complex DP as in (56) is now the complement of the preposition during. (55)(57) are very close in meaning, with (56) and (57) being basically truth-conditional equivalent.

(55) Jane wrote the article [when Jacob lived in London].

(56) Jane wrote the article [ $D P$ the year Jacob lived in London].

(57) Jane wrote the article during [ $D P$ the year Jacob lived in London].

What is crucial for us is that all three sentences in (55)-(57) exhibit the very same temporal alignment between the matrix and the embedded clauses, despite the wh-word when occurring only in (55). The matrix event of writing an article is 
On the semantics of temporal when-clauses

contained within the time interval the bracketed constituent refers to in (55)-(57) . So the $w h$-word when cannot be responsible for the temporal alignment.

It could be argued that the headed relative clause in (56) and (57) may contain a silent wh-operator identical to when, which can also occur overtly, as shown in (58) and (59).

(58) Jane wrote the article [ $D P$ the year when Jacob lived in London].

(59) Jane wrote the article during [ $D P$ the year when Jacob lived in London].

Still we have strong intuitions that it is the preposition during in (57) or (59) that marks the nature of the temporal alignment overtly. Our intuitions are supported by the fact that, if during is replaced with other prepositions like before or after, the nature of the temporal alignment changes, regardless of whether the wh-word when is present in the headed relative or not (60).

(60) Jane wrote the article before/after $[D P$ the year (when) Jacob lived in London].

We take the facts in (55)-(57) together with (60) as further support of our proposal according to which a silent preposition semantically equivalent to during takes the temporal when-clause in (55) as its complement (incidentally, we would argue the same is true for the temporal DP in (56)).

Containment is not the only temporal alignment observed between a temporal when-clause and its matrix clause. They can also be simultaneous. To see an example, it is enough to turn the aspect of the matrix predicate in (55) from perfective to imperfective/progressive, without changing anything else (61). This shows that aspectual properties of the predicate play a crucial role in determining the temporal alignment. On the other hand, the role of when seems to be null in this regard, unless when is assumed to be ambiguous between an overlapping interpretation and a simultaneous one.

(61) Mary was writing the article [when Jacob lived in London].

The very same pattern is observed with headed relative clauses with or without the wh-word when preceded or not by the preposition during (62).

(62) Mary was writing the article (during) [the year (when) Jacob lived in London].

Once again, the temporal alignment observed between a temporal when-clause and its matrix clause is found in very different constructions that do not need the wh-word when to occur. 
Finally, there are cases where the temporal when-clause and its matrix clause do not temporally overlap at all, but are contiguous, right next to each other. These are the cases where the predicates in both clauses are punctual or achievement predicates. In (63), my walking in the room (temporal when-clause) necessarily precedes my seeing Mary (matrix clause). The same holds if the temporal when-clause is replaced with a headed relative clause (64).

I saw Mary [when I walked in the room].

I saw Mary [the moment (when) I walked in the room].

The "contiguous/non-overlapping" interpretation of both (63) and (64) may be due to a "causality" implicature (e.g. I saw her because I walked into the room). Causes necessarily precede effects, therefore they cannot overlap. What is crucial for us is that temporal when-clauses allow for such flexible temporal interpretation. If the temporal alignment were built into the definition of when, then this fuzziness would need to be accounted for. If notions like "sloppy simultaneity" (Declerck 1997: 114) or "approximate coincidence" (Ritchie 1979: 90) were adopted, then the temporal alignment would be predicted to be very irregular, random, and unpredictable, which is not the pattern we have observed so far.

In conclusion, we have shown that the temporal alignment pattern between temporal when-clauses and their matrix clauses can vary from complete overlapping (simultaneity) to partial overlapping to no overlapping (continguity). This variation is sensitive to the aspectual properties of the matrix and embedded predicates. The same pattern is observed in headed relative clauses. Our proposal that the $w h$-word when does not bear responsibility for the temporal alignment is supported by these findings. Also, the assumption of a silent preposition taking the temporal whenclause as its complement is further supported by the temporal headed relative clauses preceded by an overt preposition or not that exhibit the same temporal alignment patterns.

\section{The "ambiguity" of when}

The definition of the wh-word when that we have proposed (repeated here as (65)) postulates an ambiguity. When is defined as restricting the set it applies to to "time intervals" or "events." This is not a completely unrelated ambiguity. Time intervals and events are closely related and it may even be possible that "time intervals" are subparts of "events." Events have to take place in space and time, and thus it seems natural for there to be a strong relationship between these concepts.

$$
\begin{aligned}
& \text { a. when } \sim>\lambda \mathrm{P} \lambda \mathrm{x}[\mathrm{P}(\mathrm{x}) \wedge \operatorname{time}(\mathrm{x})] \\
& \text { b. when } \sim>\lambda \mathrm{P} \lambda \mathrm{x}[\mathrm{P}(\mathrm{x}) \wedge \operatorname{event}(\mathrm{x})]
\end{aligned}
$$


On the semantics of temporal when-clauses

As the concepts are so closely related, it does not seem surprising that English uses the same word, time, for both. That is, time can either refer to a time interval (66) or to an event (67). ${ }^{3,4}$

(66) This year, the tulips bloomed at the time we all expected. (time interval)

(67) I lost my keys in the water the time I went swimming in the bay. (event)

No crucial component of our analysis hinges on whether or not this is a true ambiguity or if it can be subsumed into a more general definition of the meaning of when that unifies time intervals and events. We leave this to further investigation. Thus far, the evidence for there being a distinction has outweighed the evidence for unification, as we discuss in the remainder of this section.

Our analysis predicts the temporal when-clause in (68a) to be ambiguous between referring to a time interval and an event. This seems to match our intuitions, since both (68b) and (68c) - resulting from replacing the temporal when-clause with a relative clause headed by the nominal time - are possible paraphrases of (68a), and they clearly have different meanings. Despite their similarity, they also exhibit a difference in form. The preposition at precedes the time in (68b), while no preposition occurs in $(68 \mathrm{c})$.

a. I came to visit you [when Bill left].

b. I came to visit you $[P P$ at $[D P$ the time Bill left $]]$ (time interval)

c. I came to visit you $[D P$ the time Bill left] (event)

The difference can be further highlighted by looking at examples that allow for just one interpretation, due to their semantic content. The temporal when-clause in (69a) can only be paraphrased with the nominal time preceded by the preposition at (69b). In both sentences, the bracketed constituent can refer to the time interval. (69b) is unacceptable without the preposition, showing that the other reading is not available at all.

a. This year, the tulips bloomed [when we all expected]. (time interval)

b. This year, the tulips bloomed [*(at) the time we all expected]. (time interval)

The temporal when-clause in (70a), instead, can only be paraphrased with the nominal time without the preposition at. In both sentences, the bracketed constituent refers to the whole event of me going to swim in the bay rather than the specific time interval when that event happened.

(70) a. I lost my keys in the water [when I went swimming in the bay]. (event)

b. I lost my keys in the water [(*at) the time I went swimming in the bay]. (event)

3 See Rothstein 1995 for a detailed exploration of the event use of the word time.

4 Thanks to Orin Percus for pointing this out to us. 
Furthermore, the two uses of the word time in English are lexicalized differently in other languages. Spanish, for instance, uses a word meaning a temporal unit for the time interval meaning (la hora ' the hour/time') and a completely different lexical item for the event meaning (vez 'event/time'). The fact that other languages use different lexical items for these two uses again points at a sort of ambiguity.

Another difference in English is that the time interval reading always anchors the clause to a specific time interval, requiring the two events happen at the same time (the visiting event and the leaving event happen in the same time interval and thus happen simultaneously in (68a) under the interpretation in (68b)). The event reading, on the other hand, anchors the matrix clause to the event the when-clause refers to. Since leave in (68a) is an achievement predicate (and induces a change of state - the location of the leaver), the event reading (paraphrased in (68c) will be true as long as the visiting happens at any time such that Bill is gone - perhaps days after the actual leaving event (the exact boundaries need to be determined by contextual factors).

The behavior of the wh-word when is not idiosyncratic. English has at least two other lexical items that show similar behavior. The pro-form then, in fact, seems to range over the same two elements, time intervals and events, and the free relative introduced by the wh-word what ranges over several different entities as well. Evidence for these two claims is presented each in turn.

The pro-form then seems to be able to refer to time intervals or events. This is illustrated in (71)-(72) below where in (71) then refers to the event of going camping, not to some particular time interval, and in (72) then refers to the specific time interval at 3:45.

(71) Remember the time we went camping in the woods?

- Yeah, I had a lot of fun then.

(72) John arrived at 3:45, and I arrived then, too.

The fact that the same $w h$-word can be used to form free relatives that refer to more than one kind of object is not a unique property of the wh-word when. What, for instance, introduces free relatives whose reference ranges over at least concrete objects (73), propositions (74), and abstract objects (75).

I ate [what you cooked] concrete object

I heard [what you said] proposition

\section{I understand [what you are going through] abstract object}

The parallel between when and then and also with what further strengthens our analysis of temporal when-clauses as free relatives as it shows their behavior again patterning with other free relatives as well as their being able to serve as the antecedent of a pro-form. 
On the semantics of temporal when-clauses

In conclusion, the ambiguity we encoded in the meaning of the wh-word when between time intervals and events is aimed to capture the various syntactic/semantic facts and contrasts we just discussed. We let future investigation decide if the same result can be achieved by a more elegant unified meaning for when.

\section{Non-maximal when-clauses}

So far, we have been dealing with temporal when-clauses that refer to the maximal individual/element of a given set of time intervals or events. Still there are temporal when-clauses that exhibit a different semantic behavior. In this section, we present some examples, show that the same facts are found in free relatives introduced by where, and make a tentative suggestion on how to account for these non-maximal temporal when-clauses. Two examples of temporal when-clauses that do not refer to a maximal individual are given in (76) and (77).

(76) Call me [when you have a moment].

(77) Capt. Kirk was brave [when no one else could even fathom it].

In (76), it is clear that the speaker does not want you to call at the maximal moment (i.e. the sum of all the moments, i.e. all the moments) that you are free. Rather, it could be paraphrased as something like, "call me at a moment that you are free." Similarly, (77) does not have the interpretation that Capt. Kirk was brave at every moment that no one else could fathom being brave (this set of moments is presumably infinite), but rather that he was brave in some of those moments.

These cases of non-maximality in temporal when-clauses mirror cases of nonmaximality in free relatives introduced by the wh-word where - another piece of evidence that temporal when-clauses are free relatives. If we consider (78) and its bracketed clause, it is clear that the number of places that no one has been to is presumably infinite, and the sentence does not mean that Capt. Kirk went to all of those infinite places, but only to some.

Capt. Kirk went [where no man had gone before].

Following Caponigro (2003, 2004), we suggest that, in both the non-maximal when-clauses and non-maximal where-clauses, the appropriate interpretation is obtained through a kind interpretation (Chierchia 1998; Dayal 2004). Chierchia (1998) states that for any natural property, there should exist a corresponding kind. In his example, this would be the property of being a dog (79), which corresponds to the "dog-kind" (Chierchia 1998: 348).

Dogs bark. 
For us, the natural property in (77) would be the property of being a time that no one could fathom being brave, corresponding to the "when-no-one-could-fathom-beingbrave-kind". These properties are then type shifted via the "down" operator $\cap$ to be of type e. For simple NPs where determiners are available, type-shifting operations corresponding to those operators are "blocked", leaving the kind operator as the only available type-shifter to be available covertly. However, with free relatives, as they cannot co-occur with determiners, both type shifting operations ( $l$ and $\cap$ ) should be available and be selected by the context. We have already seen properties shifting to maximal plural individuals via $\imath$ (the canonical case), and here there appears to be the shift from property to kind via $\cap$. Note that just like the cases discussed in Chierchia 1998, not all properties can be kinds. For instance, the when-clause in (80) below cannot receive this non-maximal (kind) interpretation, presumably there are not enough instantiations of John's writing an article on astrophysics to be considered a kind.

(80) I want to go to Florida [when John is writing an article on astrophysics].

These kind NPs, can, however appear where object-level arguments appear as in Chierchia's (1998) example repeated here in (81).

Dogs are barking.

In these cases, Chierchia claims that, rather than making reference to a kind, reference is made to instantiations of a kind. This happens via "Derived Kind Predication" as described in Chierchia (1998: 364) and reported in (82).

If $\mathrm{P}$ applies to objects and $\mathrm{k}$ denotes a kind, then $\mathrm{P}(\mathrm{k})=\exists \mathrm{x}\left[{ }^{\cup} \mathrm{k}(\mathrm{x}) \wedge \mathrm{P}(\mathrm{x})\right]$

According to Chierchia (1998), the DKP is triggered by a semantic mismatch - when an object-level argument position is filled with a kind. The DKP provides existential quantification over instantiations of the kind. It could be the case that a similar process happens with free relatives, as would be expected due to their nominal status. If the when-clause makes reference to the kind "times when no one could fathom being brave", then the DKP can shift the reference to instantiations of the kind described by the when-clause.

This system for making reference to instantiations of kinds to deal with these non-maximal when-clauses appears to be on the right track. For instance, it predicts that, if there really are "moments-when-something-happens-kinds", then we would expect those phrases to occur with kind-predicates (Carlson 1977; Chierchia 1998). The utterances in (83)-(84) show that the bare plural moments, when modified by the relative clause when everyone is happy, can take a kind-predicate, be rare. This indicates that there could be a "moments-when-everyone-is-happy-kind."

[Moments when everyone is happy] are rare. 
On the semantics of temporal when-clauses

It is rare [when everyone is happy].

We have shown that temporal when-clauses can have non-maximal interpretations, much like free relatives introduced by where. This is a further example of how temporal when-clauses behave like free relatives. We have also suggested that Chierchia's Derived Kind Predication may be responsible for this non-maximal interpretation.

\section{Conclusion}

Although temporal when-clauses appear to function similarly to other embedded clauses that express a temporal relationship with the matrix clause, they are special because they are introduced by a wh-word, and yet do not have an interrogative interpretation. We have shown that the syntactic and semantic behavior of whenclauses indicates that they pattern with other non-interrogative embedded clauses introduced by $w h$-words. That is, they are free relatives, both syntactically and semantically. As such, they have the same distribution as DPs and PPs, and they can always be paraphrased with a DP or a PP. Also like other wh-clauses, the interpretation of when-clauses is sensitive to island effects, indicating the presence of a gap.

Furthermore, unlike previous semantic analyses of when, our definition does not include the temporal alignment of the matrix and the embedded clause. We have shown evidence from the pattern of temporal alignments that this information cannot be part of the meaning of when.

Our analysis is fully compatible with this as for us, the meaning of when is simply a set restrictor: it restricts sets to contain only time intervals or events. This is an improvement over previous semantic analyses that built the temporal alignment information into the definition of when.

Furthermore, our analysis makes two predictions: a crosslinguistic prediction and a processing prediction. That is, cross-linguistically, languages that have free relatives may use the same word for the interrogative and the temporal clause, and languages that do not have free relatives will use unrelated words for the two uses. Preliminarily, this prediction seems to hold: English, Italian, Danish, and Spanish have free relatives and use the same word, while Korean and Japanese do not have free relatives and use unrelated words.

The processing prediction is that since temporal when-clauses are formed through movement of the wh-word when, they should leave behind a gap. Since there has been a lot of research that focuses on the processing of filler-gap dependencies (Kluender \& Kutas 1993, among many others), then it should be possible to investigate whether temporal when-clauses show the same processing taxation that is found with other 
filler-gap dependencies. If they do, then this would provide another type of evidence that temporal when-clauses have gaps, thus supporting our analysis of these clauses as free relatives. Hall (in prep.) investigates this point.

\section{References}

Bonomi, Andrea. 1997. Aspect, quantification and when-clauses in Italian. Linguistics and Philosophy 20(5). 469-514. doi:dx.doi.org/10.1023/A:1005388230492.

Bresnan, Joan \& Jane Grimshaw. 1978. The syntax of free relatives in English. Linguistic Inquiry 9(3). 331-391. http://www.jstor.org/stable/4178069.

Caponigro, Ivano. 2003. Free Not to Ask: On the Semantics of Free Relatives and Whwords Cross-linguistically: University of California, Los Angeles dissertation.

Caponigro, Ivano. 2004. The semantic contribution of Wh-words and type shifts: Evidence from free relatives crosslinguistically. In Robert B. Young (ed.), Proceedings from Semantics and Linguistic Theory (SALT) XIV, 38-55. Ithaca, NY: CLC Publications, Cornell University.

Caponigro, Ivano \& Lisa Pearl. 2008. Silent prepositions: Evidence from free relatives. In A. Ashbury, J. Dotlacil, B. Gehrke \& R. Nouwen (eds.), The Syntax and Semantics of Spatial P, 365-385. Amsterdam: John Benjamins.

Carlson, Greg. 1977. Reference to kinds in English: UMASS, Amherst dissertation.

Chierchia, Gennaro. 1998. Reference to kinds across languages. Natural Language Semantics 6. 339-405. doi:10.1023/A:1008324218506.

Dayal, Veneeta. 2004. Number marking and (in)definiteness in kind terms. Linguistics and Philosophy 27(4). 393-350. doi:10.1023/B:LING.0000024420.80324.67.

Declerck, Renaat. 1997. When-clauses and temporal structure. New York: Routledge.

Emonds, Joseph. 1976. A transformational approach to English syntax. New York: Academic Press.

Emonds, Joseph. 1987. The invisible category principle. Linguistic Inquiry 18(4). 613-632. http://www.jstor.org/stable/4178563.

Farkas, Donca \& Yoko Sugioka. 1983. Restrictive if/when clauses. Linguistics and Philosophy 6(2). 225-258. doi:10.1007/BF00635644.

Grimshaw, Jane. 1977. English Wh-Constructions and the Theory of Grammar: UMASS, Amherst dissertation.

Hall, David P. in prep. The processing of adjunct free relatives. Manuscript: University of California, San Diego.

Jacobson, Pauline. 1995. On the quantificational force of English free relatives. In E. Bach, E. Jelinek, A. Kratzer \& B. Partee (eds.), Quantification in Natural Languages, 451-486. Dordrecht: Kluwer Academic Press. 
On the semantics of temporal when-clauses

Kluender, Robert \& Marta Kutas. 1993. Bridging the gap: Evidence from ERPs on the processing of unbounded dependencies. Journal of Cognitive Neuroscience 5(2). 196-214. doi:10.1162/jocn.1993.5.2.196.

Larson, Richard. 1985. Bare-NP Adverbs. Linguistic Inquiry 16(4). 595-621. http://www.jstor.org/stable/4178458.

Link, Godehard. 1983. The logical analysis of plural and mass terms: A latticetheoretical approach. In Rainer Bäuerle, Christoph Schwarze \& Arnim von Stechow (eds.), Meaning, Use and Interpretation of Language, Berlin: de Gruyter.

McCawley, James. 1988. Adverbial NPs: Bare or clad in see-through garb? Language 64(3). 583-590. doi:10.2307/414534.

Moens, Marc \& Mark Steedman. 1988. Temporal ontology and temporal reference. Computational Linguistics 14(2). 15-28.

van Riemsdijk, Henk. 2005. Free relatives: A syntactic case study. In Martin Everaert \& Henk van Reimsdijk (eds.), The Blackwell Companion to Syntax, Blackwell.

Ritchie, Graeme. 1979. Temporal clauses in English. Theoretical Linguistics 6(1-3). 87-115. doi:10.1515/thli.1979.6.1-3.87.

Rothstein, Susan. 1995. Adverbial quantification over events. Natural Language Semantics 3(1). 1-31. doi:10.1007/BF01252883.

Vikner, Carl. 2004. Scandinavian when clauses. Nordic Journal of Linguistics 27. 133-167.

David P. Hall

9500 Gilman Dr. \#0108

La Jolla, California 92093

dhall@ling.ucsd.edu
Ivano Caponigro

9500 Gilman Dr. \#0108

La Jolla, California 92093

ivano@ucsd.edu 